\section{SAT0303 SINGLE-CELL DECONVOLUTION OF SKIN FIBROBLAST HETEROGENEITY IN PATIENTS WITH SYSTEMIC SCLEROSIS}

Q. Yan ${ }^{1}$, R. Lii ${ }^{1}$ L. Lu' ${ }^{1}{ }^{1}$ Renji Hospital, Shanghai Jiaotong University, School of Medicine, Department of Rheumatology, Shanghai, China

Background: Fibroblast heterogeneity and homeostasis has long been recognized in patients with systemic sclerosis (SSc). However, there is no common consensus on fibroblast subtypes, lineages, biological properties, signaling, and plasticity, which severely hampers our understanding of SSc pathogenesis.

Objectives: This study is aimed to comprehensively classify skin fibroblast populations from SSc patients.

Methods: We applied single-cell RNA sequencing on skin fibroblasts from two SSc patients and two health control $(\mathrm{HC})$ with matched age and sex. Cell clustering were mainly determined by UMAP with batch effect correction. Differently expressed genes in each cell cluster was analyzed by Gene Set Enrichment Analysis (GSEA)

Results: With an unbiased approach, single-cell transcriptome analyses showed classified and defined eight fibroblast types in SSc skin and six in normal skin. The cell types seldom overlapped between the patients and HC. Extracellular interaction and collagen production were remarkably stronger in SSc fibroblasts. A subgroup of dramatic cell proliferation and activation was defined only in SSc fibroblast. Two subtypes responding inflammatory stimuli were only found in SSc patients. Furthermore, delineation of their differentiation trajectory was achieved by a machine learning method.

Conclusion: This collection of single-cell transcriptomes and the distinct classification of fibroblast subsets provide a new resource for understanding the fibroblast landscape and the roles of fibroblasts in SSc.

References: N/A

Disclosure of Interests: None declared

DOI: 10.1136/annrheumdis-2020-eular.5868

\section{SAT0304 \\ TOFACITINIB INHIBITS TGF-B1-INDUCED ACTIVATED CELL FUNCTIONS OF MYOFIBROBLAST IN HUMAN LUNG FIBROBLAST POPULATIONS}

F. Zhu' ${ }^{1}$ X. Zhang ${ }^{1}{ }^{1}$ Guangdong Provincial People's Hospital, Guangdong Academy of Medical Sciences, Department of Rheumatology, Guangzhou, China

Background: Connective tissue disease-associated interstitial lung disease (CTD-ILD) is a class of refractory diseases.Non-specific treatment with hormone and immunosuppressive agents is mostly used at present, but the effect is limited and the long-term survival rate is not improved [1], while anti-fibrosis treatments (such as Pirfenidone and Nintedanib) have only recently been approved, the long-term efficacy is still unknown.

Tofacitinib(TOFA), a JAK inhibitor, has recently been used to treat patients with severe dermatomyositis related interstitial pulmonary disease, with significantly improved survival rate [2-4].A basic study showed that TOFA improved interstitial pulmonary disease in mice by promoting the proliferation of myelogenic inhibitory cells [5]. However, whether TOFA can affect the migration and invasion of human
Control

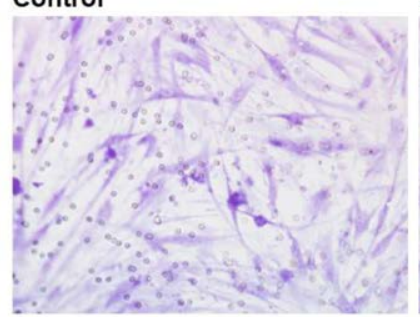

TGF- $\beta 1+$ TOFA

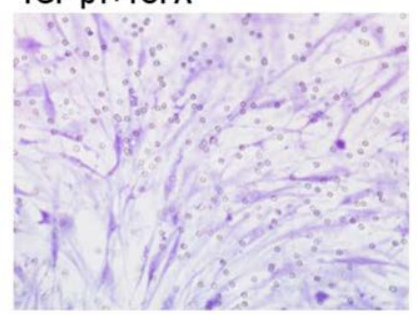

TGF- $\beta 1$
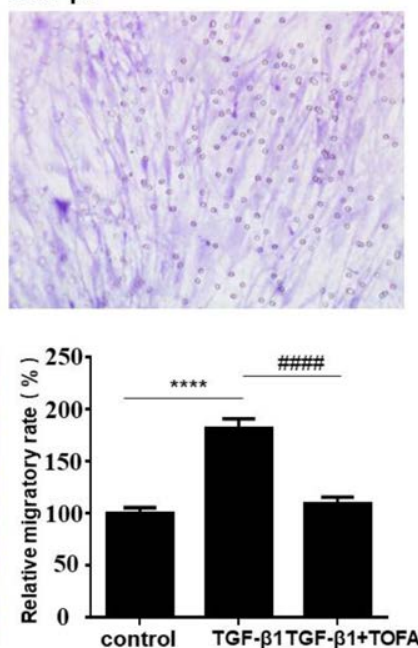

Figure 1. Effect of TOFA on HLFs migration function $(\times 200) \cdot$ Mean \pm SEM. $n=5$. lung fibroblasts and further research to reveal the mechanism of its inhibition of pulmonary fibrosis has not been reported.

Objectives: To investigate the anti - fibrosis effect of TOFA in CTD-ILD.

Methods: Cell migration and invasion Assays

HLFs were incubated with TOFA for $72 \mathrm{~h}$, followed by TGF- $\beta 1$ for $24 \mathrm{~h} . \mathrm{DMEM}$ serum-free medium was used to determine the cell density to $5.0 \times 10^{7} / \mathrm{L}, 600$ uL medium containing $10 \%$ fetal bovine serum was added to the lower compartment of Transwell chamber, and $200 \mathrm{uL}$ cell suspension was added to the upper compartment.Incubate in incubator for $12 \mathrm{~h}$.After fixation, staining and sealing, the cells were observed and counted under a microscope. At least 5 random field transmembrane cells were counted in each hole, and the mean value was taken For the invasion assays, Transwell chamber coated with matrigel was used, and the cell incubation time was $16 \mathrm{~h}$.

Results: 1. Effect of TOFA on HLFs migration function (Figure 1)

The number of cells passing through the biofilm in the three groups was counted It can be seen that TGF- $\beta 1$ group significantly increased compared with control group ( $\mathrm{P}<0.0001$ ), and TOFA group significantly decreased compared with TGF- $\beta 1$ group ( ${ }^{P} P<0.0001$ ), suggesting that TOFA can significantly inhibit TGF$\beta 1$ - induced HLFs migration.

2. Effect of TOFA on HLFs invasion function (Figure 2)

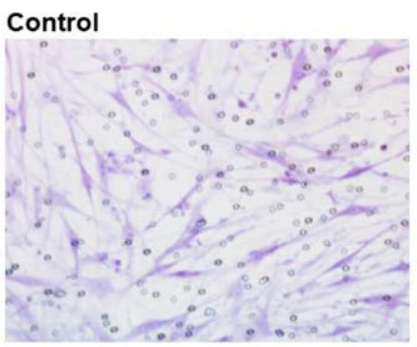

TGF- $\beta 1$

\section{TGF- $\beta 1+$ TOFA}
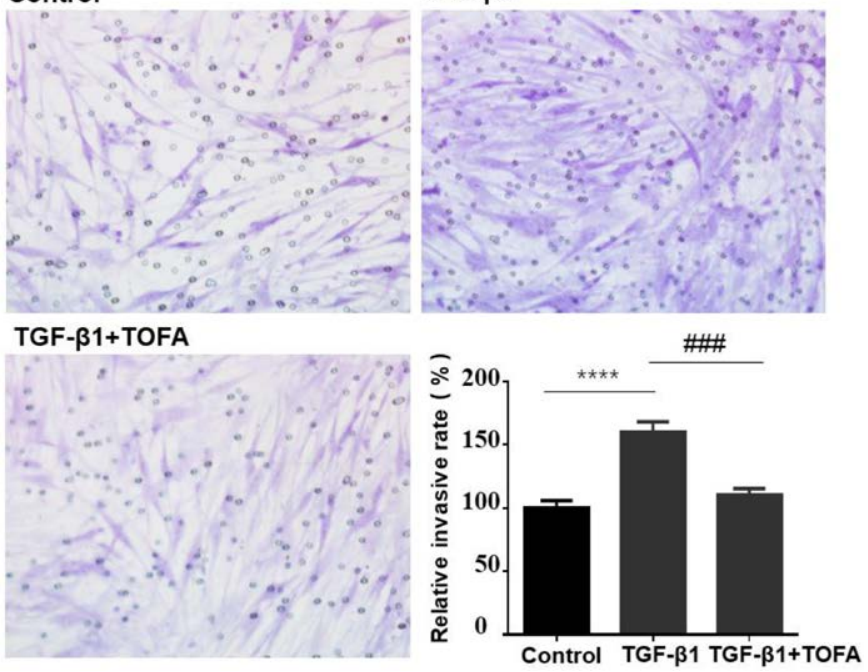

Figure 2. Effect of TOFA on HLFs invasion function $(\times 200)$.Mean \pm SEM. $n=5$

The number of cells passing through the matrigel in the three groups was counted.It can be seen that TGF- $\beta 1$ group was significantly higher than the control group ( $\mathrm{P}<0.0001$ ), and TOFA group was significantly lower than TGF- $\beta 1$ group $\left.{ }^{(\#} \mathrm{P}<0.001\right)$, suggesting that TOFA can significantly inhibit the invasion function of HLFs induced by TGF- $\beta 1$.

Conclusion: TOFA can effectively inhibit the function of HLFs migration and invasion. Although further studies are needed to elucidate the mechanism by which TOFA inhibit the function of HLFs migration and invasion, our study suggests that TOFA has a potential therapeutic effect for CTD-ILD.

\section{References:}

[1] Aparicio, I.J. and J.S. Lee, Connective Tissue Disease-Associated Interstitial Lung Diseases: Unresolved Issues. Semin Respir Crit Care Med, 2016. 37(3): p. 468-76.

[2] Kato, M., et al., Successful Treatment for Refractory Interstitial Lung Disease and Pneumomediastinum With Multidisciplinary Therapy Including Tofacitinib in a Patient With Anti-MDA5 Antibody-Positive Dermatomyositis. J Clin Rheumatol, 2019.

[3] Kurasawa, K., et al., Tofacitinib for refractory interstitial lung diseases in anti-melanoma differentiation-associated 5 gene antibody-positive dermatomyositis. Rheumatology (Oxford), 2018. 57(12): p. 2114-2119.

[4] Chen, Z., X. Wang, and S. Ye, Tofacitinib in Amyopathic Dermatomyositis-Associated Interstitial Lung Disease. N Engl J Med, 2019. 381(3): p. 291-293.

[5] Sendo, S., et al., Tofacitinib facilitates the expansion of myeloid-derived suppressor cells and ameliorates interstitial lung disease in SKG mice. Arthritis Res Ther, 2019. 21(1): p. 184

Disclosure of Interests: : None declared

DOI: 10.1136/annrheumdis-2020-eular.1139 